\title{
Learning from Massive Noisy Labeled Data for Image Classification
}

\author{
Tong Xiao ${ }^{1}$, Tian $\mathrm{Xia}^{2}$, Yi Yang ${ }^{2}$, Chang Huang ${ }^{2}$, and Xiaogang Wang ${ }^{1}$ \\ ${ }^{1}$ The Chinese University of Hong Kong \\ ${ }^{2}$ Baidu Research
}

\begin{abstract}
Large-scale supervised datasets are crucial to train convolutional neural networks (CNNs) for various computer vision problems. However, obtaining a massive amount of well-labeled data is usually very expensive and time consuming. In this paper, we introduce a general framework to train CNNs with only a limited number of clean labels and millions of easily obtained noisy labels. We model the relationships between images, class labels and label noises with a probabilistic graphical model and further integrate it into an end-to-end deep learning system. To demonstrate the effectiveness of our approach, we collect a large-scale real-world clothing classification dataset with both noisy and clean labels. Experiments on this dataset indicate that our approach can better correct the noisy labels and improves the performance of trained CNNs.
\end{abstract}

\section{Introduction}

Deep learning with large-scale supervised training dataset has recently shown very impressive improvement on multiple image recognition challenges including image classification [12], attribute learning [29], and scene classification [8]. While state-of-the-art results have been continuously reported [23, 25, 28], all these methods require reliable annotations from millions of images [6] which are often expensive and time-consuming to obtain, preventing deep models from being quickly trained on new image recognition problems. Thus it is necessary to develop new efficient labeling and training frameworks for deep learning.

One possible solution is to automatically collect a large amount of annotations from the Internet web images [10] (i.e. extracting tags from the surrounding texts or keywords from search engines) and directly use them as ground truth to train deep models. Unfortunately, these labels are extremely unreliable due to various types of noise (e.g. labeling mistakes from annotators or computing errors from extraction algorithms). Many studies have shown that these

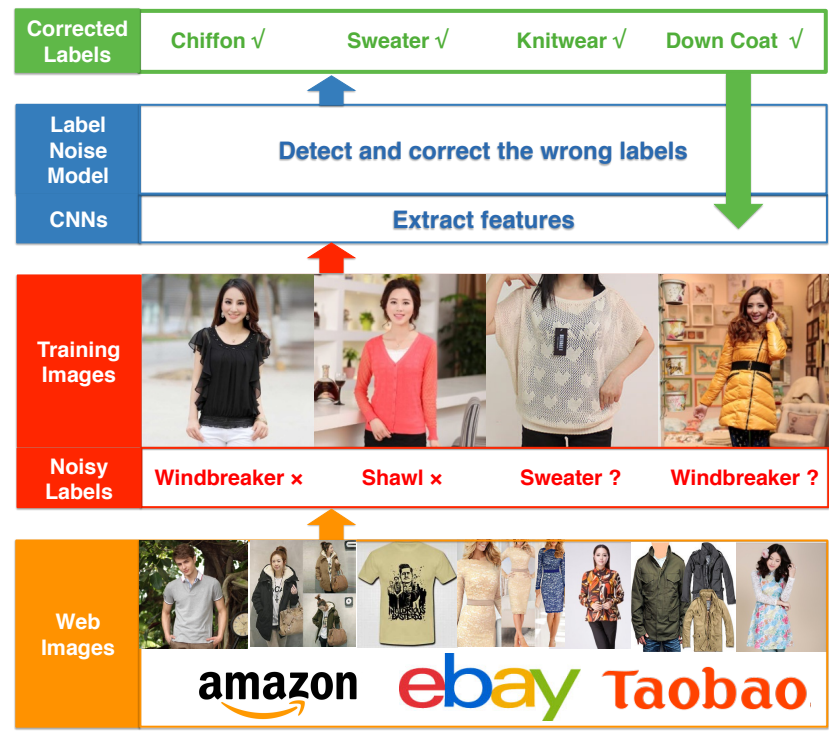

Figure 1. Overview of our approach. Labels of web images often suffer from different types of noise. A label noise model is proposed to detect and correct the wrong labels. The corrected labels are used to train underlying CNNs.

noisy labels could adversely impact the classification accuracy of the induced classifiers [20, 22, 31]. Various label noise-robust algorithms are developed but experiments show that performances of classifiers inferred by robust algorithms are still affected by label noise [3,26]. Other data cleansing algorithms are proposed [2, 5, 17], but these approaches are difficult in distinguishing informative hard examples from harmful mislabeled ones.

Although annotating all the data is costly, it is often easy to obtain a small amount of clean labels. Based on the observation of transferability of deep neural networks, people initialize parameters with a model pretrained on a larger yet related dataset [12], and then finetune on the smaller dataset of specific tasks $[1,7,21]$. Such methods may better avoid overfitting and utilize the relationships between the 
two datasets. However, we find that training a CNN from scratch with limited clean labels and massive noisy labels is better than finetuning it only on clean labels. Other approaches address the problem as semi-supervised learning where noisy labels are discarded [30]. These algorithms usually suffer from model complexity thus cannot be applied on large-scale datasets. Therefore, it is inevitable to develop a better way of using the huge amount of noisy labeled data.

Our goal is to build an end-to-end deep learning system that is capable of training with both limited clean labels and massive noisy labels more effectively. Figure 1 shows the framework of our approach. We collect 1,000, 000 clothing images from online shopping websites. Each image is automatically assigned with a noisy label according to the keywords in its surrounding text. We manually refine 72,409 image labels, which constitute a clean sub-dataset. All the data are then used to train CNNs, while the major challenge is to identify and correct wrong labels during the training process.

To cope with this challenge, we extend CNNs with a novel probabilistic model, which infers the true labels and uses them to supervise the training of the network. Our work is inspired by [24], which modifies a CNN by inserting a linear layer on top of the softmax layer to map clean labels to noisy labels. However, [24] assumes noisy labels are conditionally independent of input images given clean labels. However, when examining our collected dataset, we find that this assumption is too strong to fit the real-world data well. For example, in Figure 2, all the images should belong to "Hoodie". The top five are correct while the bottom five are either mislabeled as "Windbreaker" or "Jacket". Since different sellers have their own bias on different categories, they may provide wrong keywords for similar clothes. We observe these visual patterns and hypothesize that they are important to estimate how likely an image is mislabeled. Based on these observations, we further introduce two types of label noise:

- Confusing noise makes the noisy label reasonably wrong. It usually occurs when the image content is confusing (e.g., the samples with "?” in Figure 1).

- Pure random noise makes the noisy label totally wrong. It is often caused by either the mismatch between an image and its surrounding text, or the false conversion from the text to label (e.g., the samples with " $x$ " in Figure 1).

Our proposed probabilistic model captures the relations among images, noisy labels, ground truth labels, and noise types, where the latter two are treated as latent variables. We use the Expectation-Maximization (EM) algorithm to solve the problem and integrate it into the training process of CNNs. Experiments on our real-world clothing dataset
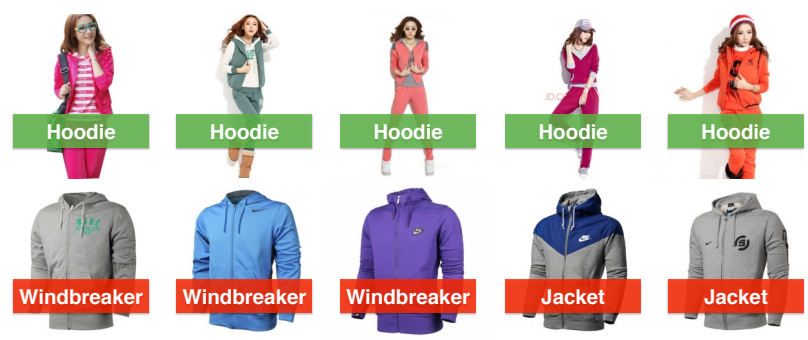

Figure 2. Mislabeled images often share similar visual patterns.

indicate that our model can better detect and correct the noisy labels.

Our contributions fall in three aspects. First, we study the cause of noisy labels in real-world data and describe it with a novel probabilistic model. Second, we integrate the model into a deep learning framework and explore different training strategies to make the CNNs learn from better supervisions. Finally, we collect a large-scale clothing dataset with both noisy and clean labels, which will be released for academic use.

\section{Related Work}

Learning with noisy labeled training data has been extensively studied in the machine learning and computer vision literature. For most of the related work including the effect of label noises, taxonomy of label noises, robust algorithms and noise cleaning algorithms for learning with noisy data, we refer to [9] for a comprehensive review.

Direct learning with noisy labels: Many studies have shown that label noises can adversely impact the classification accuracy of induced classifiers [31]. To better handle label noise, some approaches rely on training classifiers with label noise-robust algorithms [4, 15]. However, Bartlett et al. [3] prove that most of the loss functions are not completely robust to label noise. Experiments in [26] show that the classifiers inferred by label noise-robust algorithms are still affected by label noise. These methods seem to be adequate only when label noise can be safely managed by overfitting avoidance [9]. On the other hand, some label noise cleansing methods were proposed to remove or correct mislabeled instances [2,5,17], but these approaches were difficult in distinguishing informative hard examples from harmful mislabeled ones. Thus they might remove too many instances and the overcleansing could reduce the performances of classifiers [16].

Semi-supervised learning: Apart from direct learning with label noise, some semi-supervised learning algorithms were developed to utilize weakly labeled or even unlabeled data. The Label Propagation method [30] explicitly used ground truths of well labeled data to classify unlabeled samples. However, it suffered from computing pairwise dis- 
tance, which has quadratic complexity with the number of samples thus cannot be applied on large-scale datasets. Weston et al. [27] proposed to embed a pairwise loss in the middle layer of a deep neural network, which benefits the learning of discriminative features. But they needed extra information about whether a pair of unlabeled images belong to the same class, which cannot be obtained in our problem.

Transfer learning: The success of CNNs lies in their capability of learning rich and hierarchical image features. However, the model parameters cannot be properly learned when training data is not enough. Researchers proposed to conquer this problem by first initializing CNN parameters with a model pretrained on a larger yet related dataset, and then finetuning it on the smaller dataset of specific task [1, $7,12,21]$. Nevertheless, this transfer learning scheme could be suboptimal when the two tasks are just loosely related. In our case of clothing classification, we find that training a CNN from scratch with limited clean labels and massive noisy labels is better than finetuning it only on the clean labels.

Noise modeling with deep learning: Various methods have been proposed to handle label noise in different problem settings, but there are very few works about deep learning from noisy labels [13, 18, 24]. Mnih and Hinton [18] built a simple noise model for aerial images but only considered binary classification. Larsen et al. [13] assumed label noises are independent from true class labels which is a simple and special case. Sukhbaatar et al. [24] generalized from them by considering multi-class classification and modeling class dependent noise, but they assumed the noise was conditionally independent with the image content, ignoring the hardness of labeling images of different confusing levels. Our work can be viewed as a generalization of [19,24] and our model is flexible enough to not only class dependent but also image dependent noise.

\section{Label Noise Model}

We target on learning a classifier from a set of images with noisy labels. To be specific, we have a noisy labeled dataset $\mathcal{D}_{\eta}=\left\{\left(\mathbf{x}^{(1)}, \tilde{y}^{(1)}\right), \ldots,\left(\mathbf{x}^{(N)}, \tilde{y}^{(N)}\right)\right\}$ with $n$-th image $\mathbf{x}^{(n)}$ and its corresponding noisy label $\tilde{y}^{(n)} \in$ $\{1, \ldots, L\}$, where $L$ is the number of classes. We describe how the noisy label is generated by using a probabilistic graphical model shown in Figure 3.

Despite the observed image $\mathbf{x}$ and the noisy label $\tilde{\mathbf{y}}$, we exploit two discrete latent variables $-\mathbf{y}$ and $\mathbf{z}-$ to represent the true label and the label noise type, respectively. Both $\tilde{\mathbf{y}}$ and $\mathbf{y}$ are $L$-dimensional binary random variables in 1-of- $L$ fashion, i.e., only one element is equal to 1 while others are all 0.

The label noise type $\mathbf{z}$ is an 1-of-3 binary random variable. It is associated with three semantic meanings:

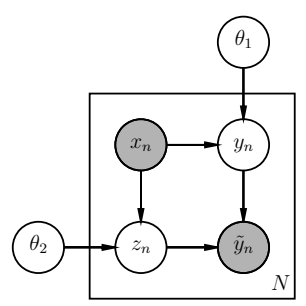

Figure 3. Probabilistic graphical model of label noise

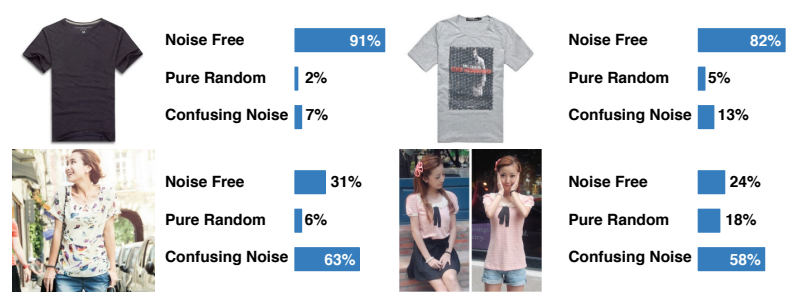

Figure 4. Predicting noise types of four different "T-shirt" images. The top two can be recognized with little ambiguity, while the bottom two are easily confusing with the class "Chiffon". Image content can affect the possibility of it to be mislabeled.

1. The label is noise free, i.e., $\tilde{\mathbf{y}}$ should be equal to $\mathbf{y}$.

2. The label suffers from a pure random noise, i.e., $\tilde{\mathbf{y}}$ can take any possible value other than $\mathbf{y}$.

3. The label suffers from a confusing noise, i.e., $\tilde{\mathbf{y}}$ can take several values that are confusing with $\mathbf{y}$.

Following this assignment rule, we define the conditional probability of the noisy label as

$$
p(\tilde{\mathbf{y}} \mid \mathbf{y}, \mathbf{z})= \begin{cases}\tilde{\mathbf{y}}^{T} \mathbf{I} \mathbf{y} & \text { if } \mathbf{z}_{1}=1 \\ \frac{1}{L-1} \tilde{\mathbf{y}}^{T}(\mathbf{U}-\mathbf{I}) \mathbf{y} & \text { if } \mathbf{z}_{2}=1 \\ \tilde{\mathbf{y}}^{T} \mathbf{C y} & \text { if } \mathbf{z}_{3}=1\end{cases}
$$

where $\mathbf{I}$ is the identity matrix, $\mathbf{U}$ is the unit matrix (all the elements are ones), $\mathbf{C}$ is a sparse stochastic matrix with $\operatorname{tr}(\mathbf{C})=0$ and $\mathbf{C}_{i j}$ denotes the confusion probability between classes $i$ and $j$. Then we can derive from Figure 3 the joint distribution of $\tilde{\mathbf{y}}, \mathbf{y}$ and $\mathbf{z}$ conditioning on $\mathbf{x}$,

$$
p(\tilde{\mathbf{y}}, \mathbf{y}, \mathbf{z} \mid \mathbf{x})=p(\tilde{\mathbf{y}} \mid \mathbf{y}, \mathbf{z}) p(\mathbf{y} \mid \mathbf{x}) p(\mathbf{z} \mid \mathbf{x})
$$

While the class label probability distribution $p(\mathbf{y} \mid \mathbf{x})$ is comprehensible, the semantic meaning of $p(\mathbf{z} \mid \mathbf{x})$ needs extra clarification: it represents how confusing the image content is. Specific to our clothing classification problem, $p(\mathbf{z} \mid \mathbf{x})$ can be affected by different factors, including background clutter, image resolution, the style and material of the clothes. Some examples are shown in Figure 4. 
To illustrate the relations between noisy and true labels, we derive their conditional probability from Eq. 2,

$$
p(\tilde{\mathbf{y}} \mid \mathbf{y}, \mathbf{x})=\sum_{\mathbf{z}} p(\tilde{\mathbf{y}}, \mathbf{z} \mid \mathbf{y}, \mathbf{x})=\sum_{\mathbf{z}} p(\tilde{\mathbf{y}} \mid \mathbf{y}, \mathbf{z}) p(\mathbf{z} \mid \mathbf{x})
$$

which can be interpreted as a mixture model. Given an input image $\mathbf{x}$, the conditional probability $p(\mathbf{z} \mid \mathbf{x})$ can be seen as the prior of each mixture component. This makes a key difference between our work and [24], where they assume $\tilde{\mathbf{y}}$ is conditionally independent with $\mathbf{x}$ if $\mathbf{y}$ is given. All the images share a same noise model in [24], while in our approach each data sample has its own.

\subsection{Learning the Parameters}

We exploit two CNNs to model $p(\mathbf{y} \mid \mathbf{x})$ and $p(\mathbf{z} \mid \mathbf{x})$ separately. Denote the parameter set of each CNN by $\theta_{1}$ and $\theta_{2}$. Our goal is to find the optimal $\theta=\theta_{1} \cup \theta_{2}$ that maximizes the incomplete $\log$-likelihood $\log p(\tilde{\mathbf{y}} \mid \mathbf{x} ; \theta)$. The EM algorithm is used to iteratively solve this problem.

For any probability distribution $q(\mathbf{y}, \mathbf{z} \mid \tilde{\mathbf{y}}, \mathbf{x})$, we can derive a lower bound of the incomplete log-likelihood,

$$
\begin{aligned}
\log p(\tilde{\mathbf{y}} \mid \mathbf{x} ; \theta) & =\log \sum_{\mathbf{y}, \mathbf{z}} p(\tilde{\mathbf{y}}, \mathbf{y}, \mathbf{z} \mid \mathbf{x} ; \theta) \\
& \geq \sum_{\mathbf{y}, \mathbf{z}} q(\mathbf{y}, \mathbf{z} \mid \tilde{\mathbf{y}}, \mathbf{x}) \log \frac{p(\tilde{\mathbf{y}}, \mathbf{y}, \mathbf{z} \mid \mathbf{x} ; \theta)}{q(\mathbf{y}, \mathbf{z} \mid \tilde{\mathbf{y}}, \mathbf{x})}
\end{aligned}
$$

E-Step The difference between $\log p(\tilde{\mathbf{y}} \mid \mathbf{x} ; \theta)$ and its lower bound is the Kullback-Leibler divergence $\operatorname{KL}(q(\mathbf{y}, \mathbf{z} \mid \tilde{\mathbf{y}}, \mathbf{x}) \| p(\mathbf{y}, \mathbf{z} \mid \tilde{\mathbf{y}}, \mathbf{x} ; \theta))$, which is equal to zero if and only if $q(\mathbf{y}, \mathbf{z} \mid \tilde{\mathbf{y}}, \mathbf{x})=p(\mathbf{y}, \mathbf{z} \mid \tilde{\mathbf{y}}, \mathbf{x} ; \theta)$. Therefore, in each iteration $t$, we first compute the posterior of latent variables using the current parameters $\theta^{(t)}$,

$$
\begin{aligned}
& p\left(\mathbf{y}, \mathbf{z} \mid \tilde{\mathbf{y}}, \mathbf{x} ; \theta^{(t)}\right)=\frac{p\left(\tilde{\mathbf{y}}, \mathbf{y}, \mathbf{z} \mid \mathbf{x} ; \theta^{(t)}\right)}{p\left(\tilde{\mathbf{y}} \mid \mathbf{x} ; \theta^{(t)}\right)} \\
& =\frac{p\left(\tilde{\mathbf{y}} \mid \mathbf{y}, \mathbf{z} ; \theta^{(t)}\right) p\left(\mathbf{y} \mid \mathbf{x} ; \theta^{(t)}\right) p\left(\mathbf{z} \mid \mathbf{x} ; \theta^{(t)}\right)}{\sum_{\mathbf{y}^{\prime}, \mathbf{z}^{\prime}} p\left(\tilde{\mathbf{y}} \mid \mathbf{y}^{\prime}, \mathbf{z}^{\prime} ; \theta^{(t)}\right) p\left(\mathbf{y}^{\prime} \mid \mathbf{x} ; \theta^{(t)}\right) p\left(\mathbf{z}^{\prime} \mid \mathbf{x} ; \theta^{(t)}\right)}
\end{aligned}
$$

Then the expected complete log-likelihood can be written as

$$
Q\left(\theta ; \theta^{(t)}\right)=\sum_{\mathbf{y}, \mathbf{z}} p\left(\mathbf{y}, \mathbf{z} \mid \tilde{\mathbf{y}}, \mathbf{x} ; \theta^{(t)}\right) \log p(\tilde{\mathbf{y}}, \mathbf{y}, \mathbf{z} \mid \mathbf{x} ; \theta)
$$

M-Step We exploit two CNNs to model the probability $p\left(\mathbf{y} \mid \mathbf{x} ; \theta_{1}\right)$ and $p\left(\mathbf{z} \mid \mathbf{x} ; \theta_{2}\right)$, respectively. Thus the gradient of $Q$ w.r.t. $\theta$ can be decoupled into two parts:

$$
\begin{array}{r}
\frac{\partial Q}{\partial \theta}=\sum_{\mathbf{y}, \mathbf{z}} p\left(\mathbf{y}, \mathbf{z} \mid \tilde{\mathbf{y}}, \mathbf{x} ; \theta^{(t)}\right) \frac{\partial}{\partial \theta} \log p(\tilde{\mathbf{y}}, \mathbf{y}, \mathbf{z} \mid \mathbf{x} ; \theta) \\
=\sum_{\mathbf{y}} p\left(\mathbf{y} \mid \tilde{\mathbf{y}}, \mathbf{x} ; \theta^{(t)}\right) \frac{\partial}{\partial \theta_{1}} \log p\left(\mathbf{y} \mid \mathbf{x} ; \theta_{1}\right)+ \\
\sum_{\mathbf{z}} p\left(\mathbf{z} \mid \tilde{\mathbf{y}}, \mathbf{x} ; \theta^{(t)}\right) \frac{\partial}{\partial \theta_{2}} \log p\left(\mathbf{z} \mid \mathbf{x} ; \theta_{2}\right) .
\end{array}
$$

The M-Step above is equivalent to minimizing the cross entropy between the estimated ground truth distribution and the prediction of the classifier.

\subsection{Estimating Matrix $\mathrm{C}$}

Notice that we do not set parameters to the conditional probability $p(\tilde{\mathbf{y}} \mid \mathbf{y}, \mathbf{z})$ in Eq. (1) and keep it unchanged during the learning process. Because without other regularizations, learning all the three parts in Eq. (2) could lead to trivial solutions. For example, the network will always predict $\mathbf{y}_{1}=1, \mathbf{z}_{3}=1$, and the matrix $\mathbf{C}$ is learned to make $\mathbf{C}_{1 i}=1$ for all $i$. To avoid such degeneration, we estimate $\mathbf{C}$ on a relatively small dataset $\mathcal{D}_{c}=\{(\mathbf{x}, \mathbf{y}, \tilde{\mathbf{y}})\}_{N}$, where we have $N$ images with both clean and noisy labels. As prior information about $\mathbf{z}$ is not available, we solve the following optimization problem:

$$
\max _{\mathbf{C}, \mathbf{z}^{(1)}, \cdots, \mathbf{z}^{(N)}} \sum_{i=1}^{N} \log p\left(\tilde{\mathbf{y}}^{(i)} \mid \mathbf{y}^{(i)}, \mathbf{z}^{(i)}\right) .
$$

Obviously, sample $i$ contributes nothing to the optimal $\mathbf{C}^{*}$ if $\mathbf{y}^{(i)}$ and $\tilde{\mathbf{y}}^{(i)}$ are equal. So that we discard those samples and reinterpret the problem in another form by exploiting Eq. (1):

$$
\max _{\mathbf{C}, \mathbf{t}} \quad E=\sum_{i=1}^{N^{\prime}} \log \alpha^{\mathbf{t}_{i}}+\log \left(\tilde{\mathbf{y}}^{(i) T} \mathbf{C y}{ }^{(i)}\right)^{1-\mathbf{t}_{i}},
$$

subject to $\mathbf{C}$ is a stochastic matrix of size $L \times L$,

$$
\mathbf{t} \in\{0,1\}^{N^{\prime}},
$$

where $\alpha=\frac{1}{L-1}$ and $N^{\prime}$ is the number of remaining samples. The semantic meaning of the above formulation is that we need to assign each $(\mathbf{y}, \tilde{\mathbf{y}})$ pair the optimal noise type, while finding the optimal $\mathbf{C}$ simultaneously.

Next, we will show that the problem can be solved by a simple yet efficient algorithm in $O\left(N^{\prime}+L^{2}\right)$ time complexity. Denote the optimal solution by $\mathbf{C}^{*}$ and $\mathbf{t}^{*}$

Theorem 1. $\mathbf{C}_{i j}^{*} \neq 0 \Rightarrow \mathbf{C}_{i j}^{*}>\alpha, \forall i, j \in\{1, \ldots, L\}$.

Proof. Suppose there exists some $i, j$ such that $0<\mathbf{C}_{i j}^{*} \leq$ $\alpha$. Then we conduct the following operations. First, we set $\mathbf{C}_{i j}^{*}=0$ while adding its original value to other elements 
in column $j$. Second, for all the samples $n$ where $\tilde{\mathbf{y}}_{i}^{(n)}=1$ and $\mathbf{y}_{j}^{(n)}=1$, we set $\mathbf{t}_{n}$ to 1 . The resulting $E$ will get increased, which leads to a contradiction.

Theorem 2. $\left(\tilde{\mathbf{y}}^{(i)}, \mathbf{y}^{(i)}\right)=\left(\tilde{\mathbf{y}}^{(j)}, \mathbf{y}^{(j)}\right) \Rightarrow \mathbf{t}_{i}^{*}=\mathbf{t}_{j}^{*}, \forall i, j \in$ $\left\{1, \ldots, N^{\prime}\right\}$.

Proof. Suppose $\tilde{\mathbf{y}}_{k}^{(i)}=\tilde{\mathbf{y}}_{k}^{(j)}=1$ and $\mathbf{y}_{l}^{(i)}=\mathbf{y}_{l}^{(j)}=1$ but $\mathbf{t}_{i}^{*} \neq \mathbf{t}_{j}^{*}$. From Theorem 1 we know that elements in $\mathbf{C}^{*}$ is either 0 or greater than $\alpha$. If $\mathbf{C}_{k l}^{*}=0$, we can set $\mathbf{t}_{i}^{*}=\mathbf{t}_{j}^{*}=1$, otherwise we can set $\mathbf{t}_{i}^{*}=\mathbf{t}_{j}^{*}=0$. In either case the resulting $E$ will get increased, which leads to a contradiction.

Theorem 3. $\tilde{\mathbf{y}}^{(i) T} \mathbf{C}^{*} \mathbf{y}^{(i)}>\alpha \Leftrightarrow \mathbf{t}_{i}^{*}=0$ and $\tilde{\mathbf{y}}^{(i) T} \mathbf{C}^{*} \mathbf{y}^{(i)}=0 \Leftrightarrow \mathbf{t}_{i}^{*}=1, \forall i \in\left\{1, \ldots, N^{\prime}\right\}$.

Proof. The first part is straightforward. For the second part, $\mathbf{t}_{i}^{*}=1$ implies $\tilde{\mathbf{y}}^{(i) T} \mathbf{C}^{*} \mathbf{y}^{(i)} \leq \alpha$. By using Theorem 1 we know that $\tilde{\mathbf{y}}^{(i) T} \mathbf{C}^{*} \mathbf{y}^{(i)}=0$.

Notice that if the true label is class $i$ while the noisy label is class $j$, then it can only affect the value of $\mathbf{C}_{i j}$. Thus each column of $\mathbf{C}$ can be optimized separately. Theorem 1 further shows that samples with same pair of $(\tilde{\mathbf{y}}, \mathbf{y})$ share a same noise type. Then what really matters is the frequencies of all the $L \times L$ pairs of $(\tilde{\mathbf{y}}, \mathbf{y})$. Considering a particular column c, suppose there are $M$ samples affecting this column. We can count the frequencies of noisy label class 1 to $L$ as $m_{1}, \ldots, m_{L}$ and might as well assume $m_{1} \geq m_{2} \geq \cdots \geq m_{L}$. The problem is then converted to

$$
\begin{array}{rl}
\max _{\mathbf{c}, \mathbf{t}} & E=\sum_{k=1}^{L} m_{k}\left(\log \alpha^{\mathbf{t}_{k}}+\log \mathbf{c}_{k}^{1-\mathbf{t}_{k}}\right), \\
\text { subject to } \quad & \mathbf{c} \in[0,1]^{L}, \sum_{k=1}^{L} \mathbf{c}_{k}=1, \\
& \mathbf{t} \in\{0,1\}^{L} .
\end{array}
$$

Due to the rearrangement inequality, we can prove that in the optimal solution,

$$
\max \left(\alpha, \mathbf{c}_{1}^{*}\right) \geq \max \left(\alpha, \mathbf{c}_{2}^{*}\right) \geq \cdots \geq \max \left(\alpha, \mathbf{c}_{L}^{*}\right) .
$$

Then by using Theorem 3, there must exist a $k^{*} \in$ $\{1, \ldots, L\}$ such that

$$
\begin{aligned}
& \mathbf{t}_{i}^{*}=0, i=1, \ldots, k^{*}, \\
& \mathbf{t}_{i}^{*}=1, i=k^{*}+1, \ldots, L .
\end{aligned}
$$

This also implies that only the first $k^{*}$ elements of $\mathbf{c}^{*}$ have nonzero values (greater than $\alpha$ actually). Furthermore, if $k^{*}$ is known, finding the optimal $\mathbf{c}^{*}$ is to solve the following problem:

$$
\begin{gathered}
\max _{\mathbf{c}} \quad E=\sum_{k=1}^{k^{*}} m_{k} \log \mathbf{c}_{k}, \\
\text { subject to } \quad \mathbf{c} \in[0,1]^{L}, \sum_{k=1}^{k^{*}} \mathbf{c}_{k}=1,
\end{gathered}
$$

whose solution is

$$
\begin{aligned}
\mathbf{c}_{i}^{*} & =\frac{m_{i}}{\sum_{k=1}^{k^{*}} m_{k}}, i=1, \ldots, k^{*}, \\
\mathbf{c}_{i}^{*} & =0, i=k^{*}+1, \ldots, L .
\end{aligned}
$$

The above analysis leads to a simple algorithm. We enumerate $k^{*}$ from 1 to $L$. For each $k^{*}, \mathbf{t}^{*}$ and $\mathbf{c}^{*}$ are computed by using Eq. (12) and (14), respectively. Then we evaluate the objective function $E$ and record the best solution.

\section{Deep Learning from Noisy Labels}

We integrate the proposed label noise model into a deep learning framework. As demonstrated in Figure 5, we predict the probability $p(\mathbf{y} \mid \mathbf{x})$ and $p(\mathbf{z} \mid \mathbf{x})$ by using two independent CNNs. Moreover, we append a label noise model layer at the end, which takes as input the CNNs' prediction scores and the observed noisy label. Stochastic Gradient Ascent with backpropagation technique is used to approximately optimize the whole network. In each forward pass, the label noise model layer computes the posterior of latent variables according to Eq. (5). While in the backward pass, it computes the gradients according to Eq. (7).

Directly training the whole network with random initialization is impractical, because the posterior computation could be totally wrong. Therefore, we need to pretrain each CNN component with strongly supervised data. Images and their ground truth labels in the dataset $\mathcal{D}_{c}$ are used to train the CNN that predicts $p(\mathbf{y} \mid \mathbf{x})$. On the other hand, the optimal solutions of $\mathbf{z}^{(1)}, \cdots, \mathbf{z}^{(N)}$ in Eq. (8) are used to train the CNN that predicts $p(\mathbf{z} \mid \mathbf{x})$.

After both CNN components are properly pretrained, we can start to train the whole network with massive noisy labeled data. However, some practical issues need further discussion. First, if we merely use noisy labels, we will lose precious knowledge that we have gained before and the model could be drifted. Therefore, we need to mix the data with clean labels into our training set, which is depicted in Figure 5 as the extra supervisions for the two CNNs. Then each CNN receives two kinds of gradients, one is from the clean labels and the other is from the noisy labels. We denote them by $\Delta_{c}$ and $\Delta_{n}$, respectively. A potential problem is that $\left|\Delta_{c}\right| \ll\left|\Delta_{n}\right|$, because clean data is much less than noisy data. To deal with this problem, we bootstrap 


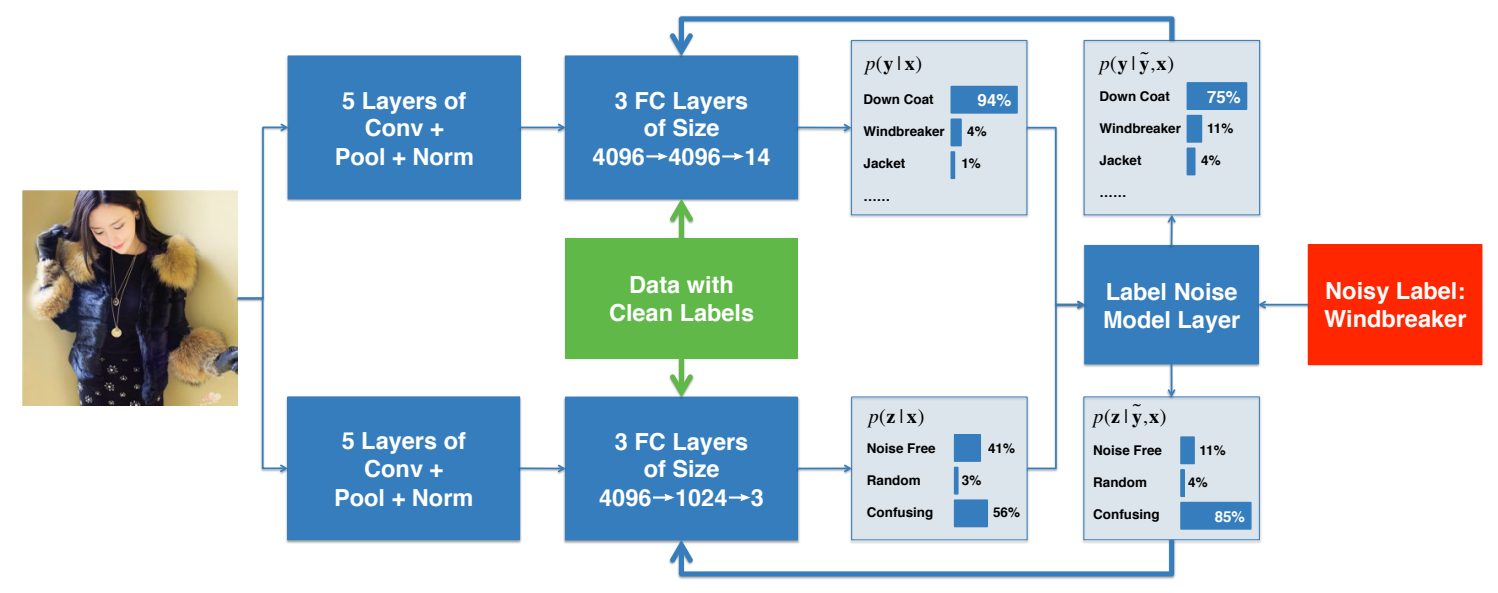

Figure 5. System diagram of our method. Two CNNs are used to predict the class label $p(\mathbf{y} \mid \mathbf{x})$ and the noise type $p(\mathbf{z} \mid \mathbf{x})$, respectively. The label noise model layer uses both these predictions and the given noisy label to estimate a posterior distribution of the true label, which is then used to supervise the training of CNNs. Data with clean labels are also mixed in to prevent the models from drifting away.

the clean data $\mathcal{D}_{c}$ to half amount of the noisy data $\mathcal{D}_{\eta}$. In our experiments, we find that the performance of the classifier drops significantly without upsampling, but it is not sensitive with the upsampling ratio as long as the number of clean and noisy samples are in the same order.

Our proposed method has the ability to figure out the true label given the image and its noisy label. From the perspective of information, our model predicts from two kinds of clues: what are the true labels for other similar images; and how likely the image is mislabeled. The Label Propagation method [30] explicitly uses the first kind of information, while we implicitly capture it with a discriminative deep model. On the other hand, the second kind of information correlates the image content with the label noise, which can help distinguish between hard samples and mislabeled ones.

\section{Experiments}

\subsection{Dataset}

Since there is no publicly available dataset that has both clean and noisy labels, to test our method under real-world scenario, we build a large-scale clothing dataset by crawling images from several online shopping websites. More than a million images are collected together with their surrounding texts provided by the sellers. These surrounding texts usually contain rich information about the products, which can be further converted to visual tags. Specific to our task of clothing classification, we define 14 class labels: T-shirt, Shirt, Knitwear, Chiffon, Sweater, Hoodie, Windbreaker, Jacket, Down Coat, Suit, Shawl, Dress, Vest, and Underwear. We assign an image a noisy label if we find its surrounding text contains only the keywords of that label, otherwise we discard the image to reduce ambiguity.
After that we manually refine the noisy labels of a small portion of all the images and split them into training $\left(\mathcal{D}_{c}\right)$, validation and test sets. On the other hand, the remaining samples construct the noisy labeled training dataset $\left(\mathcal{D}_{\eta}\right)$. A crucial step here is to remove from $\mathcal{D}_{c}$ and $\mathcal{D}_{\eta}$ the images that are near duplicate with any image in the validation or test set, which ensures the reliability of our test protocol. Finally, the size of training datasets are $\left|\mathcal{D}_{c}\right|=47,570$ and $\left|\mathcal{D}_{\eta}\right|=10^{6}$, while validation and test set have 14,313 and 10,526 images, respectively.

The confusion matrix between clean and noisy labels is presented in Figure 6. We can see that the overall accuracy is $61.54 \%$, and some pairs of classes are very confusing with each other (e.g. Knitwear and Sweater), which means that the noisy labels are not so reliable.

\subsection{Evaluation on the Collected Dataset}

We validate our method through a series of experiments conducted on the collected dataset. Our implementation is based on Caffe [11], and the bvlc_reference_caffenet ${ }^{1}$ is chosen as the baseline model, which approximates AlexNet [12]. Besides, we reimplement two other approaches. One is a semi-supervised learning method called Pseudo-Label [14], which exploits classifier's prediction as ground truth for unlabeled data. The other one is the Bottom-Up method introduced in [24], where the relation between noisy labels and clean labels are built by a confusion matrix $Q$. In the experiments, we directly use the true $Q$ as shown in Figure 6 instead of estimating its values.

We list all the experiment settings in Table 1. Different methods require different training data. We use only the clean data $\mathcal{D}_{c}$ to get the baselines under strong supervisions.

\footnotetext{
${ }^{1}$ http://caffe.berkeleyvision.org/model_zoo.html
} 


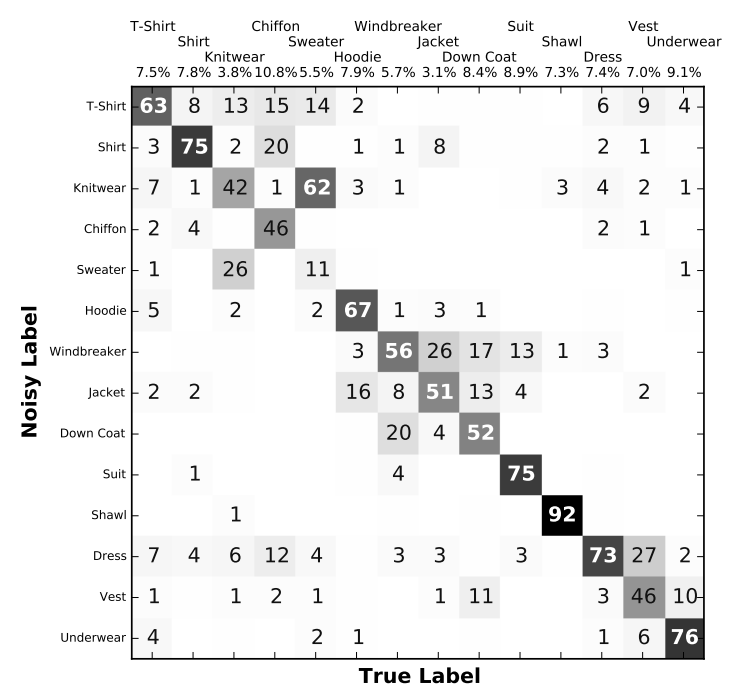

Figure 6. Confusion matrix between clean and noisy labels. We hide extremely small grid numbers for better demonstration. Frequency of each true label is listed at the top of each column. The overall accuracy is $61.54 \%$, which indicates that the noisy labels are not reliable.

On the other hand, when all the data are used, we upsample the clean data as discussed in Section 4. Meanwhile, the noisy labels of $\mathcal{D}_{\eta}$ are treated as true labels for AlexNet, and are discarded for Pseudo-Label.

In general, we use a mini-batch size of 256 . The learning rate is initialized to be 0.001 and is divided by 10 after every 50,000 iterations. We keep training each model until convergence. Classification accuracies on the test set are presented in Table 1.

We first study the effect of transfer learning and massive noisy labeled data. From row \#1 we can see that training a CNN from scratch with only small amount of clean data can result in bad performance. To deal with this problem, finetuning from an ImageNet pretrained model can significantly improve the accuracy, as shown in row \#2. However, by comparing row \#2 and \#3, we find that training with random initialization on additional massive noisy labeled data is better than finetuning only on the clean data, which demonstrates the power of using large-scale yet easily obtained noisy labeled data. The accuracy can be further improved if we finetune the model either from an ImageNet pretrained one or model \#2. The latter one has slightly better performance thus is used to initialize the remaining methods.

Next, from row \#6 we can see that semi-supervised learning methods may not be a good choice when massive noisy labeled data are available. Although model \#6 achieves marginally better result than its base model, it is

\begin{tabular}{c|c|c|c}
\hline Noise Level & CIFAR10-quick & {$[24]$} & Ours \\
\hline \hline $30 \%$ & $65.57 \%$ & $69.73 \%$ & $69.81 \%$ \\
\hline $40 \%$ & $62.38 \%$ & $66.66 \%$ & $66.76 \%$ \\
\hline $50 \%$ & $57.36 \%$ & $63.39 \%$ & $63.00 \%$ \\
\hline
\end{tabular}

Table 2. Accuracies on CIFAR-10 with synthetic label noises. The label noises generated here only depend on the true labels but not the image content, which exactly match the assumption of [24] but are unfavored to our model.

significantly inferior to model \#5, which indicates that simply discarding all the noisy labels cannot fully utilize these information.

Finally, row \#7 and \#8 show the effect of modeling the label noise. Model \#7 is only $0.9 \%$ better than the baseline model \#5, while our method gains improvement of $2.9 \%$. This result does credit to our image dependent label noise model, which fits better to the noisy labeled data crawled from the Internet.

\subsection{Evaluation on CIFAR-10 with Synthetic Noises}

We also conduct synthetic experiments on CIFAR-10 following the settings of [24]. We first randomly generate a confusion matrix $Q$ between clean labels and noisy labels, and then corrupt the training labels according to it. Based on Caffe's CIFAR10-quick model, we compare the [24] (Bottom $\mathrm{Up}$ with true $Q$ ) with our model under different noise levels. The test accuracies are reported in Table 2.

It should be noticed that [24] assumed the distribution of noisy labels only depends on classes, while we assume it also depends on image content. Label noises generated in the synthetic experiments exactly match their assumption but are unfavored to our model. Thus the noise type predictions in our model could be less informative. Nevertheless, our model achieves comparable performances with [24].

\subsection{Effect of Noise Estimation}

In order to understand how our model handles noisy labels, we first show several examples in Figure 7. We find that given a noisy label, our model exploits its current knowledge to estimate the probability distribution of the true label and then replaces the noisy one with it as supervision. Another interesting observation is that our model can still figure out the correct label if the prediction of the class label $p(\mathbf{y} \mid \mathbf{x})$ or the noise type $p(\mathbf{z} \mid \mathbf{x})$ goes wrong. These two latent variables compensate with each other to help the system distinguish between hard samples and noisy labeled ones.

Next we demonstrate the effectiveness of learning to predict the label noise type. Notice that if an image has low probability of "noise free" (i.e., $p\left(\mathbf{z}_{1}=1 \mid \mathbf{x}\right)$ is small), then our model will believe it is likely to be mislabeled. In order to check the reliability of these predictions, we estimate 


\begin{tabular}{c|c|c|c|c}
\hline$\#$ & Method & Training Data & Initialization & Test Accuracy \\
\hline \hline 1 & AlexNet & $\mathcal{D}_{c}$ & random & $64.54 \%$ \\
2 & AlexNet & $\mathcal{D}_{c}$ & ImageNet pretrained & $72.63 \%$ \\
3 & AlexNet & upsampled $\mathcal{D}_{c}$ and $\mathcal{D}_{\eta}$ as ground truths & random & $74.03 \%$ \\
4 & AlexNet & upsampled $\mathcal{D}_{c}$ and $\mathcal{D}_{\eta}$ as ground truths & ImaegNet pretrained & $75.13 \%$ \\
5 & AlexNet & upsampled $\mathcal{D}_{c}$ and $\mathcal{D}_{\eta}$ as ground truths & model \#2 & $75.30 \%$ \\
6 & Pseudo-Label [14] & upsampled $\mathcal{D}_{c}$ and $\mathcal{D}_{\eta}$ as unlabeled & model \#2 & $73.04 \%$ \\
7 & Bottom-Up [24] & upsampled $\mathcal{D}_{c}$ and $\mathcal{D}_{\eta}$ & model \#2 & $76.22 \%$ \\
8 & Ours & upsampled $\mathcal{D}_{c}$ and $\mathcal{D}_{\eta}$ & model \#2 & $\mathbf{7 8 . 2 4 \%}$ \\
\hline
\end{tabular}

Table 1. Experimental results on the clothing classification dataset. $\mathcal{D}_{c}$ contains 47,570 clean labels while $\mathcal{D}_{\eta}$ contains $10^{6}$ noisy labels.

Layout for each block

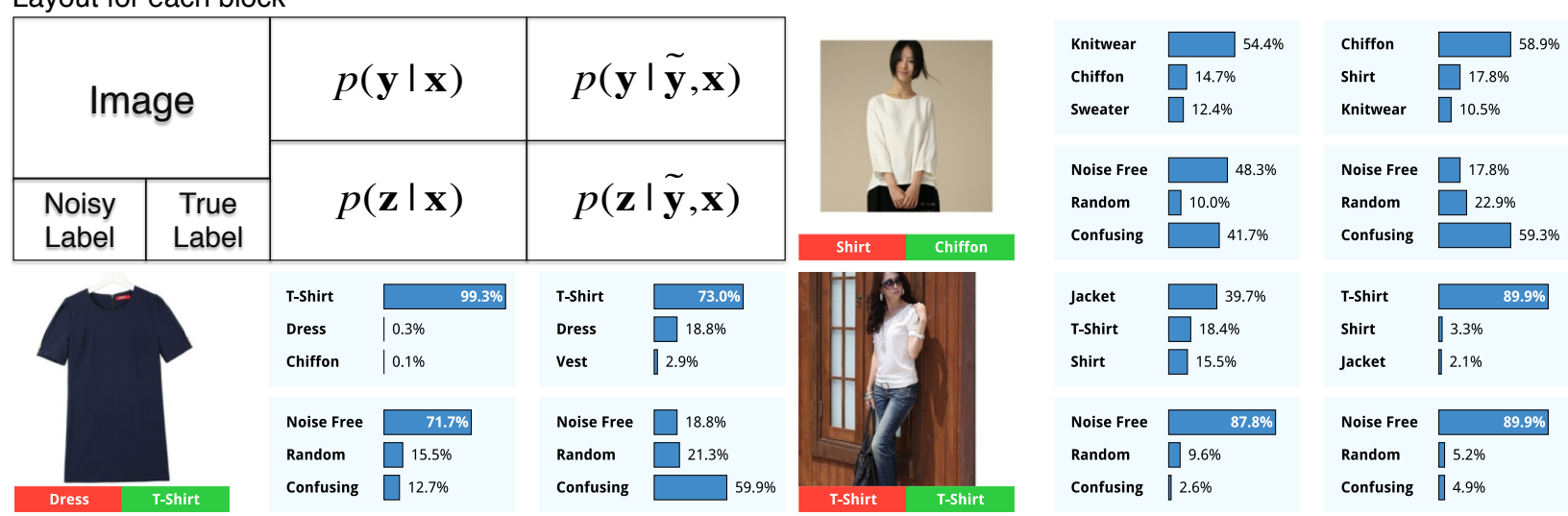

Figure 7. Examples of handling noisy labels. The information layout for each block is illustrated on the top-left. $p(\mathbf{y} \mid \mathbf{x})$ and $p(\mathbf{z} \mid \mathbf{x})$ are predictions of the true label and noise type based on image content. After observing the noisy label, our model infers the posterior distributions $p(\mathbf{y} \mid \tilde{\mathbf{y}}, \mathbf{x})$ and $p(\mathbf{z} \mid \tilde{\mathbf{y}}, \mathbf{x})$, then replaces the $\mathbf{y}$ and $\mathbf{z}$ with them as supervisions to the CNNs.

$p\left(\mathbf{z}_{1}=1 \mid \mathbf{x}\right)$ on the validation set and sort the images accordingly in ascending order. For the precision calculation, we consider a candidate image as true positive if its clean label mismatches its original noisy label, and our model predicts it as not "noisy free". The rank-precision curve is plotted in Figure 8. It shows that our model can identify mislabeled samples quite well based on their image content, which verifies the observation that mislabeled images often share similar visual patterns.

\section{Conclusion}

In this paper, we address the problem of training a deep learning classifier with a massive amount of noisy labeled training data and a small amount of clean annotations which are generally easy to collect. To utilize both limited clean labels and massive noisy labels, we propose a novel probabilistic framework to describe how noisy labels are produced. We introduce two latent variables, the true label and the noise type, to bridge the gap between an input image and its noisy label. We observe that the label noises not only depend on the ambiguity between classes, but could follow similar visual patterns as well. We build the dependency

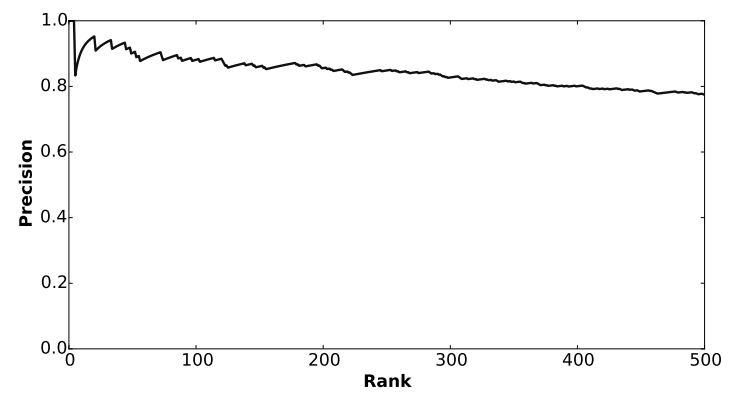

Figure 8. Rank-precision curve of label noise predictions. We rank the validation images from low to high according to their "noise free" probabilities. For the precision calculation, we consider a candidate image as true positive if its clean label mismatches its original noisy label, and our model predicts it as not "noisy free".

of the noise type w.r.t. images, and infer the ground truth label with the EM algorithm. We integrate the probabilistic model into a deep learning framework and demonstrate the effectiveness of our method on a large-scale real-world clothing classification dataset. 


\section{Acknowledgements}

This work is supported by the General Research Fund sponsored by the Research Grants Council of Hong Kong (Project Nos. CUHK14206114 and CUHK14207814) and the National Basic Research Program of China (973 program No. 2014CB340505).

\section{References}

[1] H. Azizpour, A. S. Razavian, J. Sullivan, A. Maki, and S. Carlsson. From generic to specific deep representations for visual recognition. arXiv:1406.5774, 2014. 1, 3

[2] R. Barandela and E. Gasca. Decontamination of training samples for supervised pattern recognition methods. In ICAPR. 2000. 1, 2

[3] P. L. Bartlett, M. I. Jordan, and J. D. McAuliffe. Convexity, classification, and risk bounds. Journal of the American Statistical Association, 2006. 1, 2

[4] E. Beigman and B. B. Klebanov. Learning with annotation noise. In ACL-IJCNLP, 2009. 2

[5] C. E. Brodley and M. A. Friedl. Identifying mislabeled training data. arXiv:1106.0219, 2011. 1,2

[6] J. Deng, W. Dong, R. Socher, L.-J. Li, K. Li, and L. FeiFei. Imagenet: A large-scale hierarchical image database. In CVPR, 2009. 1

[7] J. Donahue, Y. Jia, O. Vinyals, J. Hoffman, N. Zhang, E. Tzeng, and T. Darrell. Decaf: A deep convolutional activation feature for generic visual recognition. arXiv:1310.1531, 2013. 1, 3

[8] C. Farabet, C. Couprie, L. Najman, and Y. LeCun. Learning hierarchical features for scene labeling. TPAMI, 35(8):19151929, 2013. 1

[9] B. Frénay and M. Verleysen. Classification in the presence of label noise: a survey. TNNLS, 25(5):845-869, 2014. 2

[10] Y. Gong, Q. Ke, M. Isard, and S. Lazebnik. A multi-view embedding space for modeling internet images, tags, and their semantics. IJCV , 106(2):210-233, 2014. 1

[11] Y. Jia, E. Shelhamer, J. Donahue, S. Karayev, J. Long, R. Girshick, S. Guadarrama, and T. Darrell. Caffe: Convolutional architecture for fast feature embedding. In ACM MM, 2014. 6

[12] A. Krizhevsky, I. Sutskever, and G. E. Hinton. Imagenet classification with deep convolutional neural networks. In NIPS, 2012. 1, 3, 6

[13] J. Larsen, L. Nonboe, M. Hintz-Madsen, and L. K. Hansen. Design of robust neural network classifiers. In ICASSP, 1998. 3

[14] D.-H. Lee. Pseudo-label: The simple and efficient semisupervised learning method for deep neural networks. In ICML Workshop, 2013. 6, 8

[15] N. Manwani and P. Sastry. Noise tolerance under risk minimization. Cybernetics, IEEE Transactions on, 43(3):11461151, 2013. 2

[16] N. Matic, I. Guyon, L. Bottou, J. Denker, and V. Vapnik. Computer aided cleaning of large databases for character recognition. In $I A P R, 1992.2$
[17] A. L. Miranda, L. P. F. Garcia, A. C. Carvalho, and A. C. Lorena. Use of classification algorithms in noise detection and elimination. In HAIS. 2009. 1, 2

[18] V. Mnih and G. E. Hinton. Learning to label aerial images from noisy data. In ICML, 2012. 3

[19] N. Natarajan, I. S. Dhillon, P. K. Ravikumar, and A. Tewari. Learning with noisy labels. In NIPS, 2013. 3

[20] D. F. Nettleton, A. Orriols-Puig, and A. Fornells. A study of the effect of different types of noise on the precision of supervised learning techniques. Artificial intelligence review, 33(4):275-306, 2010. 1

[21] M. Oquab, L. Bottou, I. Laptev, and J. Sivic. Learning and transferring mid-level image representations using convolutional neural networks. In CVPR, 2014. 1, 3

[22] M. Pechenizkiy, A. Tsymbal, S. Puuronen, and O. Pechenizkiy. Class noise and supervised learning in medical domains: The effect of feature extraction. In CBMS, 2006. 1

[23] K. Simonyan and A. Zisserman. Very deep convolutional networks for large-scale image recognition. arXiv:1409.1556, 2014. 1

[24] S. Sukhbaatar and R. Fergus. Learning from noisy labels with deep neural networks. arXiv:1406.2080, 2014. 2, 3, 4, $6,7,8$

[25] C. Szegedy, W. Liu, Y. Jia, P. Sermanet, S. Reed, D. Anguelov, D. Erhan, V. Vanhoucke, and A. Rabinovich. Going deeper with convolutions. arXiv:1409.4842, 2014. 1

[26] C.-M. Teng. A comparison of noise handling techniques. In FLAIRS, 2001. 1, 2

[27] J. Weston, F. Ratle, H. Mobahi, and R. Collobert. Deep learning via semi-supervised embedding. In Neural Networks: Tricks of the Trade. 2012. 3

[28] M. D. Zeiler and R. Fergus. Visualizing and understanding convolutional neural networks. arXiv:1311.2901, 2013. 1

[29] N. Zhang, M. Paluri, M. Ranzato, T. Darrell, and L. Bourdev. Panda: Pose aligned networks for deep attribute modeling. In CVPR, 2014. 1

[30] X. Zhu and Z. Ghahramani. Learning from labeled and unlabeled data with label propagation. Technical report, Technical Report CMU-CALD-02-107, Carnegie Mellon University, 2002. 2, 6

[31] X. Zhu and X. Wu. Class noise vs. attribute noise: A quantitative study. Artificial Intelligence Review, 22(3):177-210, 2004. 1, 2 